\title{
Melatonin premedication versus placebo in wisdom teeth extraction: a randomised controlled trial
}

\author{
Edwin $\underline{\text { Seet }}^{1}$, MBBS, MMed, Chen Mei $\underline{\text { Liaw }}{ }^{1}$, MBBS, MMed, Sylvia $\underline{T a y^{2}}$, FDSRCS, Chang $\underline{S u}^{3}$, MS
}

INTRODUCTION Pain after wisdom teeth surgery can be moderate in severity and is compounded by preoperative anxiety in young patients. We studied the effect of melatonin premedication on postoperative pain and preoperative anxiety in patients undergoing wisdom teeth extractions.

METHODS This randomised controlled trial recruited 76 patients at Khoo Teck Puat Hospital who were American Society of Anesthesiologists physical status I and II, aged 21 to 65 and scheduled to undergo elective extraction of all four wisdom teeth under general anaesthesia. Patients with a history of long-term use or allergy to melatonin were excluded. The patients received either $6 \mathrm{mg}$ melatonin or a placebo 90 minutes before surgery. Visual analogue scale (VAS) scores at multiple time intervals for postoperative pain and preoperative anxiety, patient satisfaction and first-night sleep quality scores were obtained. Mixed-effects regression models were used for longitudinal analysis of VAS pain, anxiety and satisfaction scores.

RESULTS Maximum VAS scores for pain and anxiety were $18.6 \pm 19.1 \mathrm{~mm}$ at 60 minutes postoperatively and $26.2 \pm 23.4 \mathrm{~mm}$ at 90 minutes preoperatively, respectively. After adjusting for gender, female patients who received melatonin had a faster rate of reduction of VAS pain $(p=0.020)$ and anxiety scores $(p=0.003)$ over time compared to the placebo group. No such effect was demonstrated in male patients. There was no significant difference in sleep quality or satisfaction scores. CONCLUSION Melatonin use did not consistently contribute to pain and anxiety amelioration in all patients. Our study demonstrated a positive effect in female patients, suggestive of sexual dimorphism.

Keywords: anxiety, melatonin, pain, wisdom teeth extraction

\section{INTRODUCTION}

Wisdom teeth or third molars typically erupt into the mouth between the ages of 16 and 21 ; however, these teeth are often impacted and fail to erupt due to lack of space in the jawbones. Stagnation of food and bacteria around wisdom teeth may cause decay of the teeth and inflammation of the overlying gums, resulting in pain. A large number of wisdom teeth extraction surgeries are performed under general anaesthesia. However, research has found that postoperative pain management is generally suboptimal. ${ }^{(1)}$ In wisdom teeth extraction surgeries, this is compounded by higher levels of anxiety in young patients who may be undergoing their first operation. Anxiety has been shown to be a strong predictor of postoperative pain and analgesic consumption. ${ }^{(2)}$ It has been suggested that an anxious state lowers the pain threshold, ${ }^{(3)}$ facilitates overestimation of pain intensity ${ }^{(4)}$ and causes activation in the entorhinal cortex of the hippocampal formation. ${ }^{(5)}$

Melatonin ( $\mathrm{N}$-acetyl-5-methoxytryptamine), produced by the pineal gland, is involved in the regulation of circadian rhythms. Products containing melatonin are commonly used to alleviate jet lag and sleep disturbance. ${ }^{(6)}$ In addition, melatonin has been mooted to possess sedative, analgesic, anxiolytic, anti-inflammatory, antioxidative and chronobiotic effects. ${ }^{(7-9)}$ A qualitative systematic review of ten studies demonstrated that it was effective in decreasing preoperative anxiety as compared to a placebo, except in the elderly population. ${ }^{(10)}$ However, the included studies were heterogeneous and may have had a possible gender (female predominant) and procedural bias. The same review, which analysed qualitative data from eight trials, concluded that the analgesic effect of perioperative melatonin was controversial and warranted further investigation from well-designed randomised controlled trials. ${ }^{(10)}$ Lee, in his 2009 thesis studying 12 patients presenting for dental procedures under general anaesthesia, did not find a significant anxiolytic or sedative effect when comparing melatonin and a placebo. ${ }^{(11)}$ Isik et al also showed no significant differences in anxiolysis between melatonin and a placebo administered to children undergoing dental treatment. ${ }^{(12)}$

None of the previous published studies investigated the use of melatonin in wisdom teeth extraction surgery under general anaesthesia in a randomised controlled trial. We aim to examine the effect of melatonin premedication (90 minutes prior to surgery) on postoperative pain and preoperative anxiety in patients undergoing elective extraction of all four wisdom teeth under general anaesthesia. We hypothesise that melatonin has the potential to reduce postoperative pain and ameliorate preoperative anxiety in these patients, with a gender predilection.

\section{METHODS}

We conducted a single-centre, prospective, randomised, placebocontrolled trial funded by a Singapore National Medical Research Council grant (NMRC/NIG/1066/2011). Approval from the National Healthcare Group Domain Specific Review Board and a clinical trial certificate from the Health Sciences Authority were obtained prior to trial commencement. 
All potential subjects who presented at Khoo Teck Puat Hospital and met all the inclusion criteria were approached to participate in the study, which ran from April 2012 to July 2013. Written consent was obtained from participants by one of the investigators or a trained research nurse. The inclusion criteria for the study were: (a) American Society of Anesthesiologists physical status I to II; (b) between 21 and 65 years of age; and (c) scheduled to undergo elective extraction of all four third molar teeth under general anaesthesia. Patients with the following characteristics were excluded: history of emergent procedures, allergy to melatonin, or allergy or contraindication to drugs used in standardised anaesthesia and analgesia protocols; previously on long-term melatonin; body mass index $>35 \mathrm{~kg} / \mathrm{m}^{2}$; and serious organ disease or dysfunction, chronic pain, severe psychiatric disease or mental impairment, sleep disorders (e.g. insomnia or narcolepsy), drug addiction, pregnancy, or a language or communication barrier. The 76 patients were randomised into two groups with a 1:1 allocation using computer-generated random number codes enclosed in sealed, opaque envelopes. Treatment codes for each patient were generated by a research executive who was not participating in the study. The anaesthetists responsible for the operating room list, patients, surgeons, nurses and data collectors were all blinded to the medication and group assignment until the completion of the entire clinical trial. Group 1 patients were given melatonin $6 \mathrm{mg}$ (Nature's Farm, Singapore) and group 2 patients were given a placebo premedication 90 minutes prior to surgery. The placebo was identical in appearance to the melatonin tablets.

Patients enrolled into the study were admitted to the hospital at least two hours prior to the allocated time for surgery. Baseline visual analogue scale (VAS) scores (100 mm horizontal line, where $0=$ no symptom, and $100=$ most severe) for pain and anxiety were obtained by the research nurse. VAS scores for anxiety were reassessed 30 minutes and 60 minutes after the premedication was administered. Sedation scores ( 1 = awake, 2 = drowsy, 3 = asleep but arousable, 4 = asleep and not arousable) were assessed by the research nurse at the same time intervals. Patients received standardised general anaesthesia for surgery, according to study protocol. Induction of general anaesthesia was achieved with intravenous propofol $2-3 \mathrm{mg} / \mathrm{kg}$ and atracurium, a nondepolarising, neuromuscular-blocking agent, was administered to facilitate nasal endotracheal intubation. Anaesthesia was maintained with oxygen/air/desflurane at the discretion of the anaesthetist, with a minimum alveolar concentration of desflurane of between 0.7 and 1.2. The analgesic regime was standardised, with intravenous dexamethasone $0.1 \mathrm{mg} / \mathrm{kg}$ and intravenous fentanyl $1.5 \mathrm{mcg} / \mathrm{kg}$ at induction. Standardised dental blocks $(5.4 \mathrm{~mL} 2.0 \%$ lidocaine and $3.0 \mathrm{~mL} 0.5 \%$ bupivacaine at a proportion of three-quarters to the inferior dental nerve and one-quarter to the buccal mucosa) were administered by the oral surgeons before incision. Additional fentanyl was not allowed during the operation. After the surgery was completed, the neuromuscular blockade was reversed, followed by the removal of the endotracheal tube after the patient regained consciousness, maintained adequate ventilation and could follow commands.
Postoperatively, the patients were monitored in the postanaesthesia care unit (PACU). VAS pain scores were assessed 30 minutes, 60 minutes, 90 minutes, 120 minutes and four hours after the completion of surgery. The patients were discharged from the PACU to the day surgery ward according to their Aldrete scores and were subsequently discharged home. Discharge criteria for patients in the study were similar to those used for all patients at our institution. Postoperative sedation scores and duration in the recovery room prior to discharge to ward or home were not measured. All the patients received $1 \mathrm{~g}$ acetaminophen every 6 hours and $120 \mathrm{mg}$ etoricoxib once a day for five days after the operation. A research nurse followed up on the patients via teleconversation 24 hours after surgery to obtain VAS pain scores. Data on VAS quality of sleep ( $0=$ worst sleep and $100=$ best sleep) on the first postoperative night and the extent to which the premedication aided restful sleep was also collected. VAS patient satisfaction scores for the entire anaesthetic and surgery experience were surveyed four hours and 24 hours after surgery. The primary outcome measured was VAS pain scores 30 minutes, 60 minutes, 90 minutes, 120 minutes, four hours and 24 hours after surgery. The secondary outcomes measured were preoperative VAS anxiety scores 30 minutes and 60 minutes after the administration of premedication. VAS overall satisfaction scores at four and 24 hours after surgery, and sleep quality on the first postoperative night were also analysed.

The sample size of the study was calculated based on the null hypothesis of no difference across both groups. A previous placebo-controlled trial by Kaczmarzyk et al on analgesia in wisdom teeth operations found that a sample size of 33 patients per group was required to detect a $20 \%$ change in mean VAS pain scores after surgery (32 $\pm 6 \mathrm{~mm}$ in the control group), with an alpha-error of 0.05 and a power of $99 \% .{ }^{(13)}$ For patients with moderate postoperative pain, a minimal meaningful reduction in pain intensity corresponded to a $20 \%$ decrease in pain scores. ${ }^{(14)}$ For this study, 76 patients were recruited to take into account dropouts and protocol noncompliance. Longitudinal analysis was performed using VAS pain scores across six time points (30,60, 90 and 120 minutes, and four and 24 hours postoperatively), VAS anxiety scores across three time points (90,60 and 30 minutes preoperatively) and VAS satisfaction scores across two time points (four and 24 hours postoperatively). Mixed-effects regression models were used, incorporating random intercepts and linear trend models and adjusting for gender as a covariate. The MannWhitney $U$ test was used to compare sleep scores on the first night after surgery between the melatonin and placebo groups, and Fisher's exact test was used for binary outcomes. A p-value $<0.05$ indicated statistical significance. All the tests were conducted with Stata 13 (StataCorp, College Station, TX, USA).

\section{RESULTS}

A total of 196 patients were approached to participate in the study; 76 patients consented and were recruited. Three patients were subsequently excluded after randomisation due to immediate preoperative decisions by the surgeon to modify the surgical procedure and extract additional teeth (Fig. 1). Demographic data 


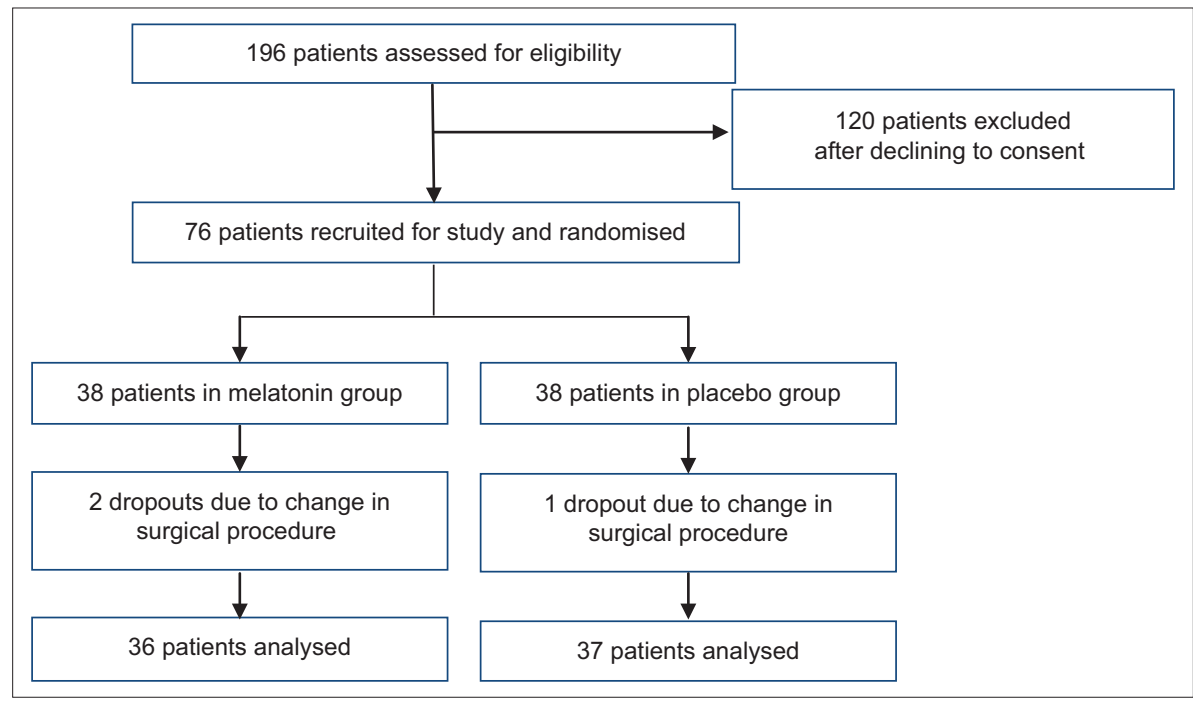

Fig. 1 CONSORT diagram shows recruitment of participants for the study.

Table I. Demographic data of the study participants $(n=73)$.

\begin{tabular}{lcc}
\hline Variable & \multicolumn{2}{c}{ Mean \pm standard deviation } \\
\cline { 2 - 3 } & $\begin{array}{c}\text { Melatonin group } \\
(\mathbf{n}=\mathbf{3 6})\end{array}$ & $\begin{array}{c}\text { Placebo group } \\
(\mathbf{n}=\mathbf{3 7})\end{array}$ \\
\hline Age (yr) & $22.7 \pm 2.2$ & $23.0 \pm 2.8$ \\
Gender* & & \\
$\quad$ Male & 24 & 23 \\
$\quad$ Female & 12 & 14 \\
Height (cm) & $167.4 \pm 8.3$ & $167.0 \pm 6.2$ \\
Weight (kg) & $65.3 \pm 14.4$ & $62.5 \pm 11.9$ \\
Body mass index (kg/m) & $22.9 \pm 3.6$ & $22.2 \pm 3.1$ \\
Surgical duration $(\mathbf{m i n})$ & $19.9 \pm 7.7$ & $21.8 \pm 10.2$ \\
Analgesia regime & & \\
$\quad$ Dexamethasone $(\mathrm{mg})$ & $6.8 \pm 1.5$ & $6.2 \pm 2.0$ \\
Fentanyl (mcg) & $96.0 \pm 23.2$ & $93.5 \pm 18.0$ \\
\hline
\end{tabular}

*Data presented as no.

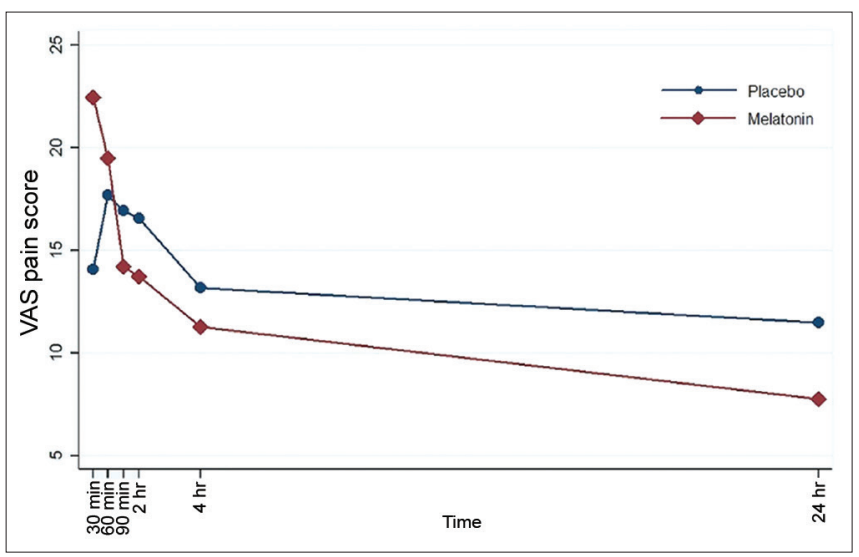

Fig. 2 Visual analogue scale (VAS) scores for postoperative pain over time for the placebo and melatonin groups. There was no significant difference between the groups.

of participants included in the study is summarised in Table I. The baseline demographic data between both groups was comparable. Due to referral to hospital from military institutions, there were more male patients than female patients in both the melatonin and placebo groups.
Table II. Patients' visual analogue scale (VAS) scores.

\begin{tabular}{|c|c|c|}
\hline \multirow[t]{2}{*}{ VAS scores (mm) } & \multicolumn{2}{|c|}{ Mean \pm standard deviation } \\
\hline & $\begin{array}{l}\text { Melatonin group } \\
\qquad(n=36)\end{array}$ & $\begin{array}{l}\text { Placebo group } \\
(n=37)\end{array}$ \\
\hline \multicolumn{3}{|l|}{ Preoperative pain } \\
\hline $90 \mathrm{~min}$ & $5.9 \pm 15.3$ & $3.1 \pm 8.6$ \\
\hline \multicolumn{3}{|l|}{ Postoperative pain } \\
\hline $30 \mathrm{~min}$ & $22.5 \pm 25.1$ & $14.1 \pm 20.7$ \\
\hline $60 \mathrm{~min}$ & $19.5 \pm 18.7$ & $17.7 \pm 19.7$ \\
\hline $90 \mathrm{~min}$ & $14.2 \pm 16.6$ & $16.9 \pm 14.7$ \\
\hline $2 \mathrm{hr}$ & $13.7 \pm 16.8$ & $16.6 \pm 16.5$ \\
\hline $4 \mathrm{hr}$ & $11.3 \pm 14.9$ & $13.2 \pm 14.9$ \\
\hline $24 \mathrm{hr}$ & $7.8 \pm 10.0$ & $11.5 \pm 13.1$ \\
\hline \multicolumn{3}{|l|}{ Preoperative anxiety } \\
\hline $90 \mathrm{~min}$ & $28.3 \pm 23.3$ & $24.2 \pm 23.7$ \\
\hline $60 \mathrm{~min}$ & $25.7 \pm 22.3$ & $23.6 \pm 24.6$ \\
\hline $30 \mathrm{~min}$ & $22.3 \pm 22.7$ & $22.7 \pm 23.8$ \\
\hline \multicolumn{3}{|l|}{ Preoperative sedation* } \\
\hline $60 \mathrm{~min}$ & $1(1-1)$ & $1(1-1)$ \\
\hline $30 \mathrm{~min}$ & $1(1-1)$ & $1(1-1)$ \\
\hline $\begin{array}{l}\text { Sleep quality on first } \\
\text { postoperative night }\end{array}$ & $82.4 \pm 20.5$ & $81.4 \pm 18.2$ \\
\hline \multicolumn{3}{|l|}{$\begin{array}{l}\text { Postoperative patient } \\
\text { satisfaction }\end{array}$} \\
\hline $4 \mathrm{hr}$ & $85.8 \pm 15.1$ & $79.2 \pm 27.0$ \\
\hline $24 \mathrm{hr}$ & $89.4 \pm 9.7$ & $88.6 \pm 13.5$ \\
\hline
\end{tabular}

*Data presented as median (interquartile range).

Preoperative sedation scores and outcome measures for VAS preoperative anxiety, postoperative pain, sleep quality on the first postoperative night and patient satisfaction are presented in Table II. Baseline preoperative pain scores in the two groups were not significantly different. Postoperative pain scores were taken at six time intervals. VAS pain scores of the melatonin group taken at 30 minutes postoperatively were not significantly different from those of the placebo group $(p=0.269)$. The patients in the melatonin group had a rate of reduction of VAS pain score that was 2.05 times faster than that of the placebo group (Fig. 2); however, this was not statistically significant $(p=0.056)$. After adjusting for gender, the decrease in VAS pain scores over time 


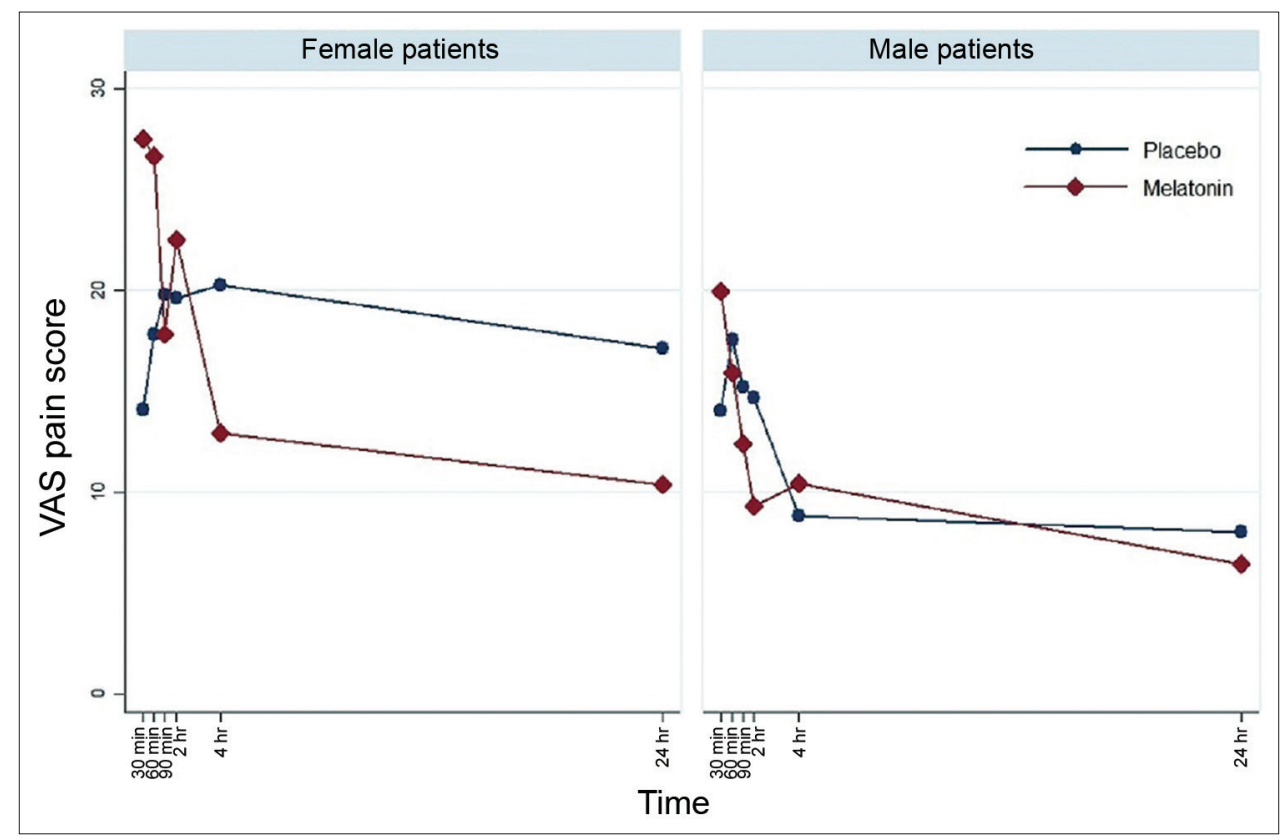

Fig. 3 Visual analogue scale (VAS) scores for postoperative pain over time by gender for the placebo and melatonin groups. VAS pain scores decreased significantly faster in the female patients.

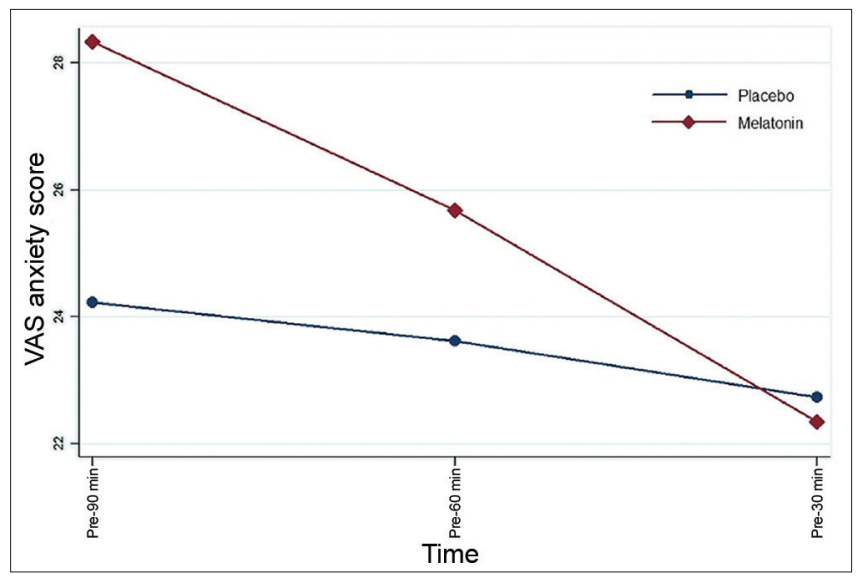

Fig. 4 Visual analogue scale (VAS) scores for preoperative anxiety over time for the placebo and melatonin groups. There was no significant difference between the groups.

was significantly faster (i.e. a difference of $4.12 \mathrm{~mm}$ ) in female patients in the melatonin group compared to those in the placebo group ( $p=0.020 ;$ Fig. 3 ). There was no significant difference in the rate of reduction of VAS pain scores between the melatonin and placebo groups for the male patients ( $p=0.509$; Fig. 3).

Preoperative anxiety scores were taken at three time intervals. Mean baseline VAS anxiety scores, taken 90 minutes before surgery, were $28.3 \pm 23.3 \mathrm{~mm}$ and $24.2 \pm 23.7 \mathrm{~mm}$ in the melatonin and placebo groups, respectively. Despite the decreasing trend for preoperative anxiety scores in the melatonin group compared to the placebo group (Fig. 4), the difference was not statistically significant $(p=0.066)$. After adjusting for gender, the rate of reduction of VAS anxiety scores over time for female patients in the melatonin group (Fig. 5) was $5.8 \mathrm{~mm}$ faster than that of the placebo group $(p=0.003)$. There was no statistical difference when comparing the rate of reduction of VAS anxiety scores over time for male patients $(p=0.835$; Fig. 5). There was no significant difference in VAS scores for first night sleep quality $(p=0.830)$ and satisfaction $(p=0.160)$ between the two groups.

Maximum VAS scores for pain and anxiety were $18.6 \pm 19.1 \mathrm{~mm}$ at 60 minutes postoperatively and $26.2 \pm 23.4 \mathrm{~mm}$ at 90 minutes preoperatively, respectively.

\section{DISCUSSION}

To the best of our knowledge, this is the first randomised placebo-controlled trial investigating the effect of melatonin on postoperative pain and preoperative anxiety in patients undergoing elective extraction of all four wisdom teeth under general anaesthesia. We found a gender-specific analgesic and anxiolytic effect of melatonin premedication in patients undergoing wisdom teeth extraction surgery.

The nature of postoperative pain differs according to the type of surgery. Recognising this, a group of surgeons and anaesthesiologists have advocated procedure-specific postoperative pain management (PROSPECT). In a recent publication, Gerbershagen et al detailed pain intensity after 179 different surgical procedures and showed that postoperative pain scores were often high and were generally worst in 'minor' surgeries due to the use of inadequate analgesics. ${ }^{(15)}$ The study showed that surgeries involving the removal or reconstruction of teeth had a moderate first-day postoperative mean pain score of 4.1 (Numeric Rating Scale 0-10). Our study yielded lower pain scores because of a standardised multimodal analgesia protocol consisting of dexamethasone, fentanyl, acetaminophen, etoricoxib and dental local anaesthesia blocks. The overall maximum VAS pain score was $18.6 \pm 19.2 \mathrm{~mm}$, experienced 60 minutes after surgery. A qualitative systematic review by Yousaf et al demonstrated that melatonin may reduce postoperative pain in certain types of surgeries - including gynaecological hysterectomies, laparoscopic cholecystectomies and cataract surgeries. ${ }^{(10)}$ Our study did not demonstrate a global 


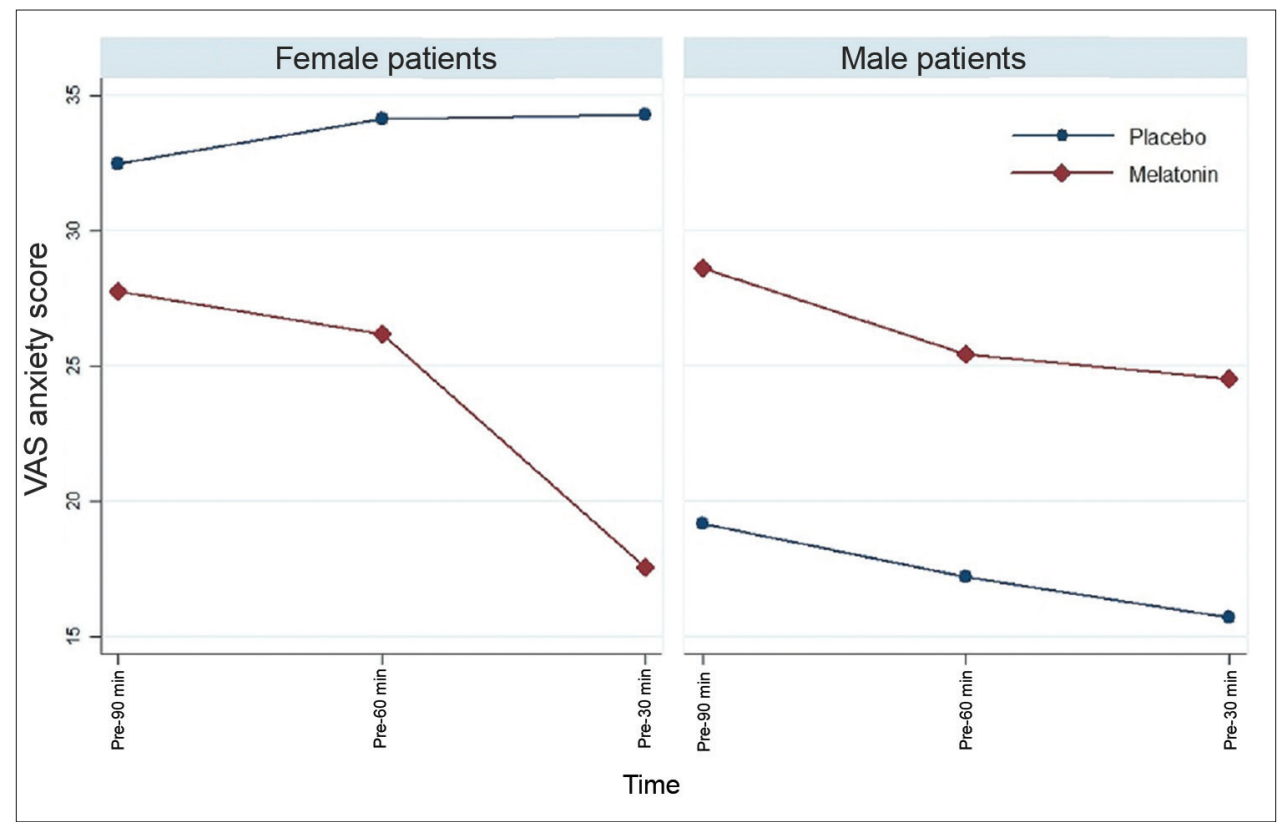

Fig. 5 Visual analogue scale (VAS) scores for preoperative anxiety over time by gender for the placebo and melatonin groups. VAS anxiety scores decreased significantly faster in the female patients.

analgesic effect relating to pain amelioration in wisdom teeth extraction $(p=0.056)$.

We used a single preoperative dose of melatonin for premedication. Andersen et al recently pointed out that the effective dosage and timing to administer melatonin are largely unknown. ${ }^{(16)}$ Recent studies performed in patients undergoing abdominal hysterectomies seemed to favour a dual-dosing regimen to reduce postoperative pain scores. ${ }^{(17-19)} \mathrm{A}$ study by Borazan et al in patients undergoing elective prostatectomy also showed a significant decrease in postoperative pain when a dual-dosing regimen was used.(20) In contrast, studies which used a once-only dosing for melatonin did not show a significant difference in pain scores when compared with a placebo. ${ }^{(21-23)}$ However, Andersen et al also noted in a recent review article that other factors, including dosage used, administration route and timing of melatonin administration prior to surgery, may affect the effects of melatonin and that further studies were required to demonstrate the dose-response relationship for melatonin. ${ }^{(24)}$ Despite a paucity of evidence for the overall benefit of melatonin in ameliorating postoperative pain in wisdom teeth extraction surgery, we have uncovered the interesting finding that after adjusting for gender, female patients who were given melatonin demonstrated faster pain reduction over time compared to those given a placebo $(p=0.020)$. The analgesic effect of melatonin in male patients was found to be absent. This finding is consistent with that of previous studies investigating pain outcomes with melatonin in female-only populations undergoing surgery. ${ }^{(17,18)}$

Baseline preoperative VAS anxiety scores 90 minutes prior to surgery were found to be $26.2 \pm 23.4 \mathrm{~mm}$ in our study population. Preoperative melatonin use as an anxiolytic agent with minimal side effects has been well-documented. ${ }^{(10,16,24)}$ Similar to the results from Lee's thesis, ${ }^{(11)}$ our study did not demonstrate a global effect; melatonin did not contribute significantly to reducing anxiety in all patients undergoing wisdom teeth extraction $(p=0.066)$. Contrary to previous publications, our study did not show preoperative anxiety to be significantly higher in female patients compared to male patients. ${ }^{(25,26)}$ Most of the current available literature studying the effects of melatonin in reducing preoperative anxiety consisted of female patients undergoing gynaecological procedures or laparoscopic cholecystectomies. ${ }^{(10)}$ Following multivariate analysis, we again found that melatonin had a gender-specific effect; female patients who received melatonin premedication had a significantly faster rate of anxiety reduction than those who received a placebo $(p=0.003)$. Our results concur with studies by Caumo et $\mathrm{al}^{(17,18)}$ as well as Naguib et al, ${ }^{(22,23)}$ which showed the benefits of melatonin as an anxiolytic agent in gynaecological surgeries. Postoperative sleep patterns can be altered by surgery and anaesthesia, which affect the normal circadian pattern of melatonin production. ${ }^{(27)}$ Previous studies have shown slight improvements in sleep quality in the early postoperative period when patients were given melatonin compared to a placebo. ${ }^{(18,20,28)}$ However, our study did not demonstrate a significant difference in sleep quality between both groups $(p=0.830)$.

This is the first known hypothesis-generating, randomised, placebo-controlled trial in dental surgery that demonstrates possible sexual dimorphism for melatonin as an analgesic and anxiolytic premedicant. Although our study had a larger sample size than other similar randomised-controlled trials, it may be important to note that the number of patients in this study was relatively small. This may have resulted in a Type II error due to insufficient power to show a difference in gender effects. Studies with female-only populations have previously shown significant benefits in terms of reduction in pain and anxiety; ${ }^{(17,18,22,23)}$ however, none of them compared the effects of melatonin use in male versus female patients. Further randomised-controlled trials may be able to shed more light on this issue. 
Based on observations from earlier clinical trials, a review article on the use of perioperative melatonin postulated that it had clinical efficacy in a narrow, select group of anxious patients with high anticipated postoperative pain. ${ }^{(10)}$ A dual-dosing regimen with an added night-before-surgery dose of melatonin was suggested to be favourable. ${ }^{(10)}$ However, we were unable to institute a dual-dosing regimen for the present study as all the patients were scheduled for ambulatory surgery. Another limitation of the study was that a protocolised multimodal analgesic regime resulted in low maximum pain scores after surgery in our study population. Therefore, we only observed a small change in absolute VAS pain scores, although the effect of melatonin was found to be significant compared to the placebo in female patients. Lastly, despite randomisation, the placebo group had higher (not statistically significant) baseline postoperative pain and preoperative anxiety scores.

In conclusion, postoperative VAS pain scores after wisdom teeth extraction surgery under general anaesthesia were generally low in our study, in part due to multimodal analgesia techniques. Despite evidence supporting the efficacy of melatonin in other populations such as gynaecological surgery patients, melatonin use did not consistently contribute to pain and anxiety amelioration in all our patients presenting for extraction of four wisdom teeth under general anaesthesia. A positive analgesic and anxiolytic effect of melatonin was demonstrated in female patients but not male patients. Female patients may benefit from melatonin premedication; however, future studies are required to elucidate plausible causes for sexual dimorphism and determine procedurespecific analgesic effects of melatonin in the perioperative period.

\section{ACKNOWLEDGEMENTS}

We would like to thank the Director of the Clinical Research Unit, Associate Professor Lim Su Chi, for his guidance and Research Nurse Ms Liew Yiting for her assistance in conducting the study.

\section{REFERENCES}

1. Warfield CA, Kahn CH. Acute pain management. Programs in U.S. hospitals and experiences and attitudes among U.S. adults. Anesthesiology 1995; 83:1090-4.

2. Ip HY, Abrishami A, Peng PW, Wong J, Chung F. Predictors of postoperative pain and analgesic consumption: a qualitative systematic review. Anesthesiology 2009; 111:657-77.

3. Rhudy JL, Meagher MW. Fear and anxiety: divergent effects on human pain thresholds. Pain 2000; 84:65-75

4. al Absi M, Rokke PD. Can anxiety help us tolerate pain? Pain 1991; 46:43-51.

5. Ploghaus A, Narain C, Beckmann CF, et al. Exacerbation of pain by anxiety is associated with activity in a hippocampal network. J Neurosci 2001; 21:9896-903.

6. Wetterberg L. Melatonin and clinical application. Reprod Nutr Dev 1999; 39:367-82.

7. Naguib M, Gottumukkala V, Goldstein PA. Melatonin and anesthesia: a clinical perspective. J Pineal Res 2007; 42:12-21

8. Arendt J, Skene DJ. Melatonin as a chronobiotic. Sleep Med Rev 2005; 9:25-39.

9. Gitto E, Romeo C, Reiter RJ, et al. Melatonin reduces oxidative stress in surgical neonates. J Pediatr Surg 2004; 39:184-9.

10. Yousaf F, Seet E, Venkatraghavan L, Abrishami A, Chung F. Efficacy and safety of melatonin as an anxiolytic and analgesic in the perioperative period: a qualitative systematic review of randomized trials. Anesthesiology 2010; 113:968-76.

11. Lee D. A study of melatonin for premedication prior to anesthesia (thesis). Toronto: University of Toronto, 2009.

12. Isik B, Baygin $\mathrm{O}$, Bodur $\mathrm{H}$. Premedication with melatonin vs midazolam in anxious children. Paediatr Anaesth 2008; 18:635-41.

13. Kaczmarzyk T, Wichlinski J, Stypulkowska J, Zaleska M, Woron J. Preemptive effect of ketoprofen on postoperative pain following third molar surgery. A prospective, randomized, double-blinded clinical trial. Int J Oral Maxillofac Surg 2010; 39:647-52.

14. Cepeda MS, Africano JM, Polo R, Alcala R, Carr DB. What decline in pain intensity is meaningful to patients with acute pain? Pain 2003; 105:151-7.

15. Gerbershagen HJ, Aduckathil S, van Wijck AJ, et al. Pain intensity on the first day after surgery: a prospective cohort study comparing 179 surgical procedures. Anesthesiology 2013; 118:934-44.

16. Andersen LP, Rosenberg J, Gögenur I. Perioperative melatonin: not ready for prime time. Br J Anaesth 2014; 112:7-8.

17. Caumo W, Levandovski R, Hidalgo MP. Preoperative anxiolytic effect of melatonin and clonidine on postoperative pain and morphine consumption in patients undergoing abdominal hysterectomy: a double-blind, randomized, placebo-controlled study. J Pain 2009; 10:100-8.

18. Caumo W, Torres F, Moreira NL Jr, et al. The clinical impact of preoperative melatonin on postoperative outcomes in patients undergoing abdominal hysterectomy. Anesth Analg 2007; 105:1263-71.

19. Ionescu D, Bãdescu C, Ilie A, et al. Melatonin as premedication for laparoscopic cholecystectomy: a double-blind, placebo-controlled study. South Afr J Anaesth Analg 2008; 14:8-11.

20. Borazan H, Tuncer S, Yalcin N, Erol A, Otelcioglu S. Effects of preoperative oral melatonin medication on postoperative analgesia, sleep quality, and sedation in patients undergoing elective prostatectomy: a randomized clinical trial. J Anesth 2010; 24:155-60.

21. Acil M, Basgul E, Celiker V, et al. Perioperative effects of melatonin and midazolam premedication on sedation, orientation, anxiety scores and psychomotor performance. Eur J Anaesthesiol 2004; 21:553-7.

22. Naguib M, Samarkandi AH. Premedication with melatonin: a doubleblind, placebo-controlled comparison with midazolam. Br J Anaesth 1999; 82:875-80.

23. Naguib M, Samarkandi AH, Moniem MA, et al. The effects of melatonin premedication on propofol and thiopental induction dose-response curves: a prospective, randomized, double-blind study. Anesth Analg 2006; 103:1448-52.

24. Andersen LP, Werner MU, Rosenberg J, Gögenur I. A systematic review of peri-operative melatonin. Anaesthesia 2014; 69:1163-71.

25. Mavridou P, Dimitriou V, Manataki A, Arnaoutoglou E, Papadopoulos G. Patient's anxiety and fear of anesthesia: effect of gender, age, education, and previous experience of anesthesia. A survey of 400 patients. J Anesth 2013; 27:104-8.

26. Mitchell M. Anaesthesia type, gender and anxiety. J Perioper Pract 2013; 23:41-7.

27. Kärkelä J, Vakkuri O, Kaukinen S, Huang WQ, Pasanen M. The influence of anaesthesia and surgery on the circadian rhythm of melatonin. Acta Anaesthesiol Scand 2002; 46:30-6.

28. Gögenur I, Kücükakin B, Bisgaard T, et al. The effect of melatonin on sleep quality after laparoscopic cholecystectomy: a randomized, placebocontrolled trial. Anesth Analg 2009; 108:1152-6. 